\title{
Surviving stress in pancreatic cancer
}

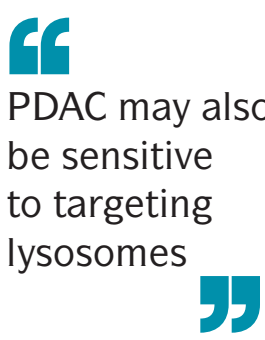

Perera et al. investigated the high levels of autophagy in pancreatic ductal adenocarcinoma (PDAC).

Autophagy involves the formation of autophagosomes, which fuse with lysosomes that in turn lyse the contents, providing building blocks for use within the cell. The authors assessed both autophagosomes and lysosomes using immunohistochemistry and found that both organelles were significantly larger in PDAC cell lines than in controls. Moreover, transmission electron microscopy showed that the number of lysosomes was higher in PDAC samples than in normal pancreatic tissue.

To assess how autophagosomes and lysosomes are increased in size and number in PDAC, the authors carried out gene-set enrichment analysis of multiple datasets and found that human PDACs have increased expression of autophagy and lysosome genes compared with normal pancreatic tissue. Lysosome and autophagosome biogenesis is regulated by the MiT/TFE family of transcription factors. Indeed, compared with 10 common solid tumour types, PDAC had the third highest
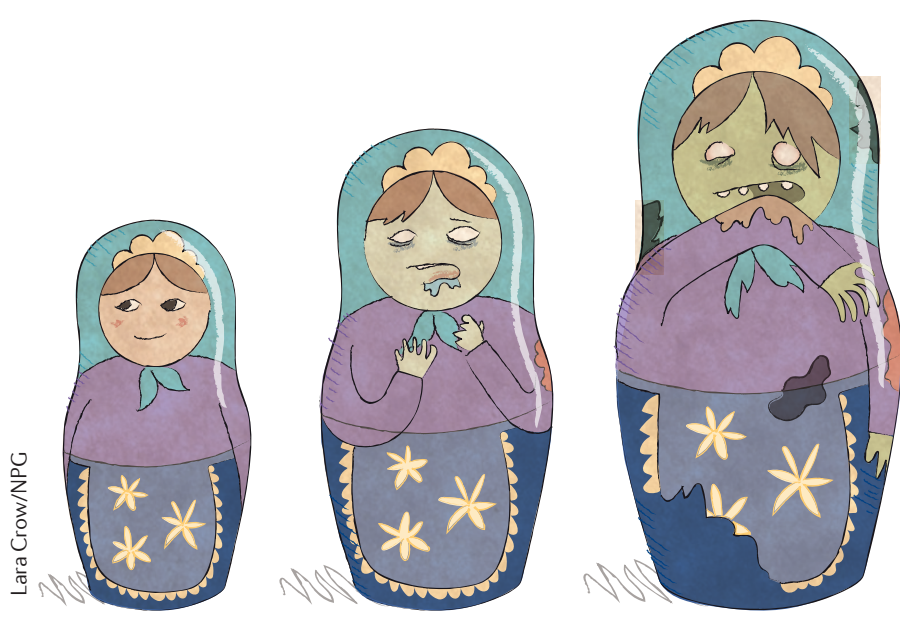

levels of MiT/TFE factors (after melanoma and kidney cancers, which are known to be associated with these factors). Further analyses of PDAC samples and cell lines revealed that a subset had high levels of individual MiT/TFE family members, especially TFE3, TFEB and MITF. The increased expression of MiT/TFE factors correlated with the increased size of autophagosomes and lysosomes in PDAC. Furthermore, knockdown of TFE3, TFEB or MITF in PDAC cell lines with high levels of these factors caused the downregulation of autophagy- and lysosome-associated target genes.

The PDAC cell lines overexpressing MiT/TFE factors also showed constitutive nuclear localization of TFE3, TFEB and MITF (an indication of their activation), even when mTOR complex 1 (mTORC1) - which mediates the cytoplasmic retention of MiT/TFE factors - was activated. The authors investigated what leads to this escape from negative regulation in PDAC cells. They found that the nucleocytoplasmic transporter importin 8 (IPO8) was enriched in TFE3 immunoprecipitates from PDAC cells (but not from normal pancreatic epithelial cells). Knockdown of IPO8 and IPO7

decreased the nuclear accumulation of TFE3, TFEB and MITF in PDAC cell lines.

Having established that knockdown of these MiT/TFE factors in PDAC cells changed lysosome morphology, the authors showed that the lysosomes were also dysfunctional. Specifically, they found that TFE3 knockdown impaired lysosomal proteolysis of extracellular factors taken up through macropinocytosis. Therefore, MiT/TFE factors promote the breakdown of molecules delivered to lysosomes by autophagy and macropinocytosis, which could provide a source of nutrients to PDAC cells. Indeed, metabolite profiling in the absence of TFE3 revealed a prominent decrease in the levels of amino acids and their breakdown products. They also showed that PDAC cells overexpressing TFE3 or MITF were able to sustain mTORC1 activity and had increased clonogenic growth compared with controls when cultured in low amino acid medium. This indicates that increased autophagy and lysosome activity allow PDAC cells to tolerate conditions of reduced amino acids.

The authors showed that knockdown of TFE3, TFEB or MITF specifically impaired the growth of PDAC cells that overexpressed each factor in culture or as xenografts, whereas controls were unaffected. Moreover, MITF overexpression considerably increased the tumorigenicity of mouse pancreatic epithelial cells expressing KRAS-G12D that were orthotopically injected into recipient mice.

Therefore, increased autophagy and lysosomal catabolism seem to be hallmarks of PDAC that provide metabolic endurance in nutrient-poor (especially low amino acid) conditions. Indeed, the authors showed that pancreatic epithelial cells overexpressing TFE3 or MITF were particularly sensitive to chloroquine, which inhibits lysosome activity, suggesting that PDAC may also be sensitive to agents targeting lysosomes.

Gemma K. Alderton

ORIGINAL RESEARCH PAPER Perera, R. M. et al

Transcriptional control of autophagy-lysosome function drives pancreatic cancer metabolism. Nature http:/dx.doi.org/10.1038/nature14587 (2015) 